\title{
Analysis of fuel using the Direct LSC method determination of bio-originated fuel in the presence of quenching
}

Charles G. Doll*, Cherylyn W. Wright, Shannon M. Morley, Bob W. Wright

\begin{abstract}
A modified version of the Direct LSC method to correct for quenching effect was investigated for the determination of bio-originated fuel content in fuel samples produced from multiple biological starting materials. The modified method was found to be accurate in determining the percent bio-originated fuel to within $5 \%$ of the actual value for samples with quenching effects $\leq 43 \%$. Analysis of highly quenched samples was possible when diluted with the exception of one sample with a $100 \%$ quenching effect.
\end{abstract}

Keywords: LSC, bio-originated fuel, biofuel, renewable fuel, C-14, quenching

\section{Introduction}

Introduction of federal tax subsidies for bio-originated fuels has created the potential of representing fossil fuel as counterfeit bio-based fuel in order to illegitimately collect subsidies or tax credits. For example, renewable diesel created from hydrotreating (refinery based hydrogenation) fats and oils to remove metals, oxygen, and nitrogen become similar to petroleum-based diesel and meet the same specifications. To thwart the counterfeiting of bio-originated fuels, methods to determine the presence or absence of bio-originated fuel in a sample are of interest. The absence of ${ }^{14} \mathrm{C}$ in fossil fuel due to decay creates a signature that can be exploited by Liquid Scintillation Counting (LSC) for determination of bio-originated fuel in a mixed sample. Therefore, LSC can provide a simple method of evaluating biooriginated fuel content. The Direct LSC method has been outlined in studies for the determination of bio-originated fuel composition (Perkin Elmer, 2009; Dijs et. al., 2006; Kristof et al, 2013; Kristof et al, 2014, Idoeta et. al., 2014; Norton et. al., 2012) and was investigated as a method of distinguishing bio-originated fuel and petroleum based fuels.

While the Direct LSC method has promise as a simple method to distinguish between biooriginated and petroleum based fuels, quenching effects create a problem for some samples resulting in lower LSC efficiency relative to unquenched samples. This quenching is a result of either chemical or color interference in the solution that reduces the light emitted from the LSC cocktail (Minne et. al., 2009; Kristof et.al., 2011; Pawlyta, et.al., 1998; Varlam et.al., 2001). To allow for the analysis of quenched samples, a method to analyze and correct for quenching in diverse fuel samples is required. We have developed a Modified Direct LSC method which incorporates internal spiking along with sample preparation techniques for the 
determination of the percent bio-originated fuel content in samples which exhibit varying amounts of quenching.

\section{Experimental}

\subsection{Materials}

Chemicals used in this study were: toluene obtained from Fisher Scientific, Ultima Gold F liquid scintillation cocktail obtained from Perkin Elmer and a ${ }^{14} \mathrm{C}-\mathrm{n}$-hexadecane certified standard with a radioactive concentration of $5.402 \times 10^{4} \mathrm{~Bq} / \mathrm{g}$ purchased from National Institute of Standards and Technology. In addition, Glass pipettes and $20 \mathrm{~mL}$ glass LSC vials with foil lids were used for all sample analysis to avoid any potential leaching of organics from plastic into the fuel. Fuel samples used in this study are listed in Table 1 and Table 2. The "Bio" samples of Table 2 are biodiesels that were determined to have less than approximately $0.1 \%$ diesel blending by gas chromatography (GC/FID) analysis; they all meet ASTM D6751 requirements for Grade No. 1-B or Grade No. 2-B biodiesel for parameters tested. The "Renewable" samples of Table 2 are homogeneous hydrocarbon oils as determined by gas chromatography/mass spectrometry (GC/MS) analysis; they both meet ASTM D975 requirements for No. 2-D diesel fuel oil for parameters tested.

Table 1. Fuels used for standard curve

\begin{tabular}{|l|l|l|l|}
\hline Sample Name & Composition & Starting Material & Description \\
\hline Renewable Blend 1 & $\begin{array}{l}\text { Bio-originated fuel } \\
\text { blend of Renewable } \\
\text { fuel from two } \\
\text { sources }\end{array}$ & $\begin{array}{l}\text { Renewable feedstock } \\
\text { originating from } \\
\text { biological sources }\end{array}$ & $\begin{array}{l}\text { Renewable that } \\
\text { meets ASTM D975 } \\
\text { requirements for } \\
\text { Grade No. 2-D diesel } \\
\text { fuel oil for parameters } \\
\text { tested }\end{array}$ \\
\hline Fossil 1 & Petroleum Diesel & $\begin{array}{l}\text { Diesel fuel from } \\
\text { Chevron Phillips } \\
\text { Chemical Company, } \\
\text { LP, SRF High Cetane } \\
\text { Check Fuel Lot } \\
\text { 2BPCFH01 }\end{array}$ & Used for Standards \\
\hline Fossil 2 & Petroleum Diesel & $\begin{array}{l}\text { DF21502 Diesel fuel } \\
\text { received as part of } \\
\text { ASTM Proficiency } \\
\text { Testing Program }\end{array}$ & Dilution Sample \\
\hline
\end{tabular}


Table 2. Samples analyzed

\begin{tabular}{|c|c|c|c|}
\hline Sample & Composition & Starting Materiala & Color \\
\hline Bio 1 & $\begin{array}{l}100 \% \text { Bio-originated } \\
\text { fuel }\end{array}$ & Unknown & Light amber \\
\hline Bio 2 & $\begin{array}{l}100 \% \text { Bio-originated } \\
\text { fuel }\end{array}$ & $\begin{array}{l}\text { Soy or other vegetable } \\
\text { based oil }\end{array}$ & Pale yellow \\
\hline Bio 3 & $\begin{array}{l}100 \% \text { Bio-originated } \\
\text { fuel }\end{array}$ & Beef tallow & Deep yellow \\
\hline Bio 4 & $\begin{array}{l}100 \% \text { Bio-originated } \\
\text { fuel }\end{array}$ & $\begin{array}{l}\text { Soy or other vegetable } \\
\text { based oil* }\end{array}$ & Clear \\
\hline Bio 5 & $\begin{array}{l}100 \% \text { Bio-originated } \\
\text { fuel }\end{array}$ & $\begin{array}{l}\text { Soy or other vegetable } \\
\text { based oil* }\end{array}$ & Pale yellow \\
\hline Bio 6 & $\begin{array}{l}100 \% \text { Bio-originated } \\
\text { fuel }\end{array}$ & Animal based fat* & Very pale yellow \\
\hline Bio 7 & $\begin{array}{l}100 \% \text { Bio-originated } \\
\text { fuel }\end{array}$ & Corn oil & Deep orange \\
\hline Bio 8 & $\begin{array}{l}100 \% \text { Bio-originated } \\
\text { fuel }\end{array}$ & Camelina oil & Yellow \\
\hline Bio 9 & $\begin{array}{l}100 \% \text { Bio-originated } \\
\text { fuel }\end{array}$ & Palm heart oil & Yellow \\
\hline Bio 10 & $\begin{array}{l}100 \% \text { Bio-originated } \\
\text { fuel }\end{array}$ & $\begin{array}{l}\text { Soy or other vegetable } \\
\text { based oil }{ }^{*}\end{array}$ & Brownish amber \\
\hline Bio 11 & $\begin{array}{l}100 \% \text { Bio-originated } \\
\text { fuel }\end{array}$ & $\begin{array}{l}\text { Soy or other vegetable } \\
\text { based oil }{ }^{*}\end{array}$ & Deep yellow \\
\hline $\begin{array}{l}\text { Renewable } \\
1\end{array}$ & $\begin{array}{l}\text { 100\% Renewable } \\
\text { Fuel }\end{array}$ & $\begin{array}{l}\text { Renewable feedstock } \\
\text { originating from biological } \\
\text { sources }\end{array}$ & Clear \\
\hline $\begin{array}{l}\text { Renewable } \\
2\end{array}$ & $\begin{array}{l}\text { 100\% Renewable } \\
\text { Fuel }\end{array}$ & $\begin{array}{l}\text { Renewable feedstock } \\
\text { originating from biological } \\
\text { sources }\end{array}$ & Clear \\
\hline
\end{tabular}

aStarting materials marked with an * are based on GC/FID analysis; others are based on information provided with the sample.

\subsection{LSC Analyses}

Sample analysis was performed on a Perkin Elmer Quantulus 1220 Low Level Liquid Scintillation Spectrometer. The instrument parameters were set to 60 or 300 minute counting 
time, ${ }^{14} \mathrm{C}$ high energy configuration, high coincidence bias, PAC setting of 1 and channel window of $50-650$.

\subsection{Spiking Solution Preparation}

Serial dilution of the ${ }^{14} \mathrm{C}$-n-hexadecane standard in toluene was performed to prepare a 2.5 $\mathrm{dpm} / \mu \mathrm{L}$ spiking standard solution.

\subsection{Standard and Sample Preparation}

Standards were prepared by mixing weighted aliquots of $100 \%$ bio-originated fuel (Renewable Blend 1) and 100\% fossil fuel (Fossil 2) in glass LSC vials for a total of $8 \mathrm{~mL}$. Fuels used for preparation of the standard curve are listed in Table 1. Prior to analysis, $12 \mathrm{~mL}$ of Ultama Gold $\mathrm{F}$ was added to each vial. For the standard curve, unspiked standards containing 0, 3.8, $8.8,12.5,16.3,21.3,25.0,37.5,50.0$, and $100.0 \%$ bio-originated fuel were prepared. After counting these unspiked standards, spiked standards were prepared and counted by adding $40 \mu \mathrm{l}$ of the $2.5 \mathrm{dpm} / \mu \mathrm{L}$ spiking standard solution ( $100 \mathrm{dpm}$ total spike) to each vial.

Samples were prepared in the same manner as for the standard curve with $8 \mathrm{~mL}$ of $100 \%$ or diluted bio-originated fuel - fossil fuel was used to dilute sample when preparing $10 \%$ and $20 \%$ bo-originated fuel samples. Spiked samples were prepared in the same manner as the spiked standards.

\subsection{Sample Cleanup}

Sample cleanup of highly quenched and dyed bio-originated fuel was achieved by passing the sample through a Carbon/ $\mathrm{NH}_{2}$ solid phase extraction (SPE) tubes (Mega BE, Agilent Technologies) using a plunger at approximately $4 \mathrm{~mL} / \mathrm{min}$. The fuel was added to a fresh SPE tube in 4-mL increments. To avoid sample dilution, no eluent was used. The sample was analyzed by GC/FID prior to and after cleanup.

\subsection{Determination of Counting Efficiency and Percent Bio-originated fuel}

By comparing the expected contribution of the spiking solution to the actual differenced spiked and unspiked samples, the percent quenching for each sample was determined along with the quenching factor.

After correcting for background, this was used to calculate a quench corrected CPM values for each sample: 
Samples were efficiency corrected and compared to standard curve to determine the percent bio-originated fuel in the unknown sample.

\section{Results and Discussion}

\subsection{Preparation of Standard Curve}

A standard curve was prepared by mixing varying amounts of $100 \%$ bio-originated fuel and petroleum fuel which were uncolored to avoid quenching effects. This series of samples contained $0,3.8,8.8,12.5,16.3,21.3,25.0,37.5,50.0$, and $100.0 \%$ bio-originated fuel. The fuels used for the standard curve and samples are listed in Table 1. After the initial analysis by LSC the standards were spiked with a ${ }^{14} \mathrm{C}$ internal standard and reanalyzed to correct for any potential quenching effects.

\subsection{Determination of Standard Stability and Optimal LSC Counting}

The standard curve samples were stored at room temperature in the dark and analyzed at 4, 40, 60 and 120 days after preparation to determine their stability over time. The samples were found to within standard deviation for time points up to 120 (data not shown).

The effect of LSC counting time on data precision was also investigated to determine the required counting time. Samples were analyzed for 60 and 300 minutes. The statistical error is approximately double for the samples when analyzed for 60 minutes compared to 300 minutes - the standard curve for both counting times are very similar. Therefore, for many applications a 60 minute count time would be adequate (data not shown).

\subsection{Sample Analysis}

For the initial investigation, eleven samples were selected for analysis based on variations in color, sampling location and provided starting material information (or likely starting material based on GC/FID profile) along with two renewable bio-originated fuel products from varying sources that had passed ASTM D975 for No.2-D S15 diesel fuel standards for parameters tested. Additional sample information is listed in Table 2. All analyzed samples contained $100 \%$ bio-originated fuel and were prepared without dilution - these samples are the $100 \%$ Fuel samples. Figure 1 compares the color differences between these samples. After the initial LSC analysis of the samples, an internal ${ }^{14} \mathrm{C}$ standard was added to each sample and analysis was repeated - these samples are the "Spiked" 100\% Fuel samples.

\section{Analysis of $100 \%$ Fuel Samples}

While it was possible to calculate the bio-originated fuel content in some of the $100 \%$ Fuel samples that had little or no quenching effect, the results were generally unsatisfactory. To correct for quenching, a quenching factor based on the internal ${ }^{14} \mathrm{C}$ spike was calculated (Table 3 ) and used to determine the quench corrected cpm and percent bio-originated fuel for each sample (Table 4). This approach was successful in determining the percent bio-originated 
fuels to within $5 \%$ for all samples with quenching values up to $43 \%$. Samples with quenching values greater than $90 \%$ had inaccurate results. It should be noted that no samples analyzed in this study had quenching values between $43-90 \%$.

Table 3. Quenching factors calculated for $100 \%$ Fuel samples. Several of the samples had $>90 \%$ quenching.

\begin{tabular}{l|rr|rr|rr|r} 
& \multicolumn{2}{|c|}{$\begin{array}{c}\text { Unspiked } \\
\text { Samples }\end{array}$} & \multicolumn{2}{|c|}{ Spiked Samples } & & \\
Sample & CPM & Error & CPM & Error & $\begin{array}{c}\text { Quenchin } \\
\mathbf{g}\end{array}$ & Error & $\begin{array}{c}\text { Quenching } \\
\text { Factor }\end{array}$ \\
\hline $100 \%$ Bio 1 & 6.47 & $5.13 \%$ & 13.13 & $3.60 \%$ & $91.79 \%$ & $9 \%$ & 0.08 \\
$100 \%$ Bio 2 & 55.04 & $1.76 \%$ & 130.26 & $1.14 \%$ & $7.27 \%$ & $4 \%$ & 0.93 \\
$100 \%$ Bio 3 & 31.62 & $2.32 \%$ & 77.82 & $1.48 \%$ & $43.05 \%$ & $5 \%$ & 0.57 \\
$100 \%$ Bio 4 & 58.54 & $1.71 \%$ & 140.72 & $1.10 \%$ & $-1.31 \%$ & $4 \%$ & 1.01 \\
$100 \%$ Bio 5 & 50.10 & $1.84 \%$ & 119.24 & $1.20 \%$ & $14.77 \%$ & $4 \%$ & 0.85 \\
$100 \%$ Bio 6 & 54.94 & $1.76 \%$ & 135.49 & $1.12 \%$ & $0.70 \%$ & $4 \%$ & 0.99 \\
$100 \%$ Bio 7 & 0.17 & $31.62 \%$ & 0.13 & $35.36 \%$ & $100.05 \%$ & $177 \%$ & 0.00 \\
$100 \%$ Bio 8 & 34.63 & $2.22 \%$ & 83.90 & $1.42 \%$ & $39.26 \%$ & $5 \%$ & 0.61 \\
$100 \%$ Bio 9 & 43.73 & $1.97 \%$ & 106.32 & $1.27 \%$ & $22.84 \%$ & $4 \%$ & 0.77 \\
$100 \%$ Bio 10 & 3.59 & $6.88 \%$ & 6.57 & $5.09 \%$ & $96.33 \%$ & $14 \%$ & 0.04 \\
$100 \%$ Bio 11 & 2.35 & $8.51 \%$ & 2.84 & $7.74 \%$ & $99.40 \%$ & $61 \%$ & 0.01 \\
$100 \%$ Renewable 1 & 56.79 & $0.77 \%$ & 141.48 & $0.49 \%$ & $-3.05 \%$ & $4 \%$ & 1.03 \\
$100 \%$ Renewable 2 & 58.89 & $0.76 \%$ & 144.53 & $0.49 \%$ & $-4.21 \%$ & $4 \%$ & 1.04
\end{tabular}

Table 4. Quench corrected Bio-originated fuel content of the 100\% Fuel samples. Note: Results for samples with quenching values $>90 \%$ were not accurate. 


\begin{tabular}{l|rc|cc|rr} 
& \multicolumn{2}{|c|}{$\begin{array}{c}\text { Quench } \\
\text { corrected }\end{array}$} & \multicolumn{2}{c|}{$\begin{array}{c}\text { Efficiency } \\
\text { Corrected }\end{array}$} & \multicolumn{2}{c}{ Calculated Results } \\
Sample & CPM & \pm CPM & DPM & \pm DPM & $\begin{array}{c}\% \text { Bio- } \\
\text { originated }\end{array}$ & Error \\
\hline $100 \%$ Bio 1 & 127.16 & $10.63 \%$ & 158.36 & $10.71 \%$ & 86.5 & $10.7 \%$ \\
$100 \%$ Bio 2 & 137.58 & $4.49 \%$ & 171.33 & $4.65 \%$ & 105.4 & $4.7 \%$ \\
$100 \%$ Bio 3 & 131.92 & $4.94 \%$ & 164.29 & $5.09 \%$ & 95.1 & $5.1 \%$ \\
$100 \%$ Bio 4 & 136.25 & $4.42 \%$ & 169.68 & $4.59 \%$ & 103.0 & $4.6 \%$ \\
$100 \%$ Bio 5 & 136.74 & $4.56 \%$ & 170.30 & $4.72 \%$ & 103.9 & $4.7 \%$ \\
$100 \%$ Bio 6 & 133.74 & $4.42 \%$ & 166.56 & $4.59 \%$ & 98.4 & $4.6 \%$ \\
$100 \%$ Bio 7 & 5191.68 & $177.07 \%$ & 6465.61 & $177.07 \%$ & 9286.1 & $177.1 \%$ \\
$100 \%$ Bio 8 & 133.71 & $4.87 \%$ & 166.52 & $5.02 \%$ & 98.4 & $5.0 \%$ \\
$100 \%$ Bio 9 & 134.31 & $4.63 \%$ & 167.27 & $4.80 \%$ & 99.5 & $4.8 \%$ \\
$100 \%$ Bio 10 & 105.62 & $17.68 \%$ & 131.54 & $17.72 \%$ & 47.3 & $17.7 \%$ \\
$100 \%$ Bio 11 & 24.83 & $213.41 \%$ & 30.93 & $213.41 \%$ & -99.4 & $213.4 \%$ \\
$100 \%$ Renewable 1 & 135.09 & $3.79 \%$ & 168.24 & $3.99 \%$ & 100.9 & $4.0 \%$ \\
$100 \%$ Renewable 2 & 136.52 & $3.80 \%$ & 170.02 & $3.99 \%$ & 103.5 & $4.0 \%$ \\
& & & & & & \\
\hline
\end{tabular}

\section{$20 \%$ Diluted Fuel Samples}

To determine the effect of sample concentration on quenching effects, 10 samples were prepared by diluting the bio-originated fuel samples to $20 \%$ with petroleum fuel - these are the "20\% Diluted Fuel" samples. The two renewable and the Bio 4 Fuel samples were not re-analyzed as dilute samples because there was little or no quenching in these clear samples. The determined quenching factors are tabulated in Table 5 and the bio-originated fuel content in Table 6. Quenching was reduced in the diluted compared to the $100 \%$ Fuel samples and they were determined to be within $4.2 \%$ of the expected $20 \%$ bio-originated fuel for all samples but the $20 \%$ Bio 7 sample which was still completely quenched and unquantifiable when diluted.

Table 5. Quenching factor calculated for $20 \%$ Diluted Fuel samples.

\begin{tabular}{c|cc|cc|cc|c} 
& \multicolumn{2}{|c|}{ Diluted Samples } & \multicolumn{2}{|c|}{ Spiked Samples } & & & \\
Sample & CPM 1 & Error & CPM 1 & Error & $\begin{array}{c}\text { Quenchin } \\
\mathbf{g}\end{array}$ & Error & $\begin{array}{c}\text { Quenching } \\
\text { Factor }\end{array}$ \\
\hline $20 \%$ Bio 1 & 8.74 & $1.97 \%$ & 60.26 & $0.75 \%$ & $37.31 \%$ & $4 \%$ & 0.63
\end{tabular}




\begin{tabular}{l|cc|cc|cc|c}
$20 \%$ Bio 2 & 11.85 & $1.70 \%$ & 84.23 & $0.64 \%$ & $11.93 \%$ & $4 \%$ & 0.88 \\
$20 \%$ Bio 3 & 7.99 & $2.06 \%$ & 72.32 & $0.69 \%$ & $21.72 \%$ & $4 \%$ & 0.78 \\
$20 \%$ Bio 5 & 11.08 & $1.75 \%$ & 78.78 & $0.66 \%$ & $17.62 \%$ & $4 \%$ & 0.82 \\
$20 \%$ Bio 6 & 11.13 & $1.75 \%$ & 80.43 & $0.65 \%$ & $15.67 \%$ & $4 \%$ & 0.84 \\
$20 \%$ Bio 7 & 0.84 & $6.36 \%$ & 0.79 & $6.54 \%$ & $100.06 \%$ & $149 \%$ & 0.00 \\
$20 \%$ Bio 8 & 10.58 & $1.79 \%$ & 78.19 & $0.66 \%$ & $17.73 \%$ & $4 \%$ & 0.82 \\
$20 \%$ Bio 9 & 10.54 & $1.80 \%$ & 78.83 & $0.66 \%$ & $16.90 \%$ & $4 \%$ & 0.83 \\
$20 \%$ Bio 10 & 7.15 & $2.18 \%$ & 62.25 & $0.74 \%$ & $32.95 \%$ & $4 \%$ & 0.67 \\
$20 \%$ Bio 11 & 7.02 & $2.20 \%$ & 42.05 & $0.90 \%$ & $57.37 \%$ & $4 \%$ & 0.43
\end{tabular}

Table 6. Quench corrected Bio-originated fuel content of the $20 \%$ Fuel samples.

\begin{tabular}{|c|c|c|c|c|c|c|}
\hline \multirow[b]{2}{*}{ Sample } & \multicolumn{2}{|c|}{$\begin{array}{l}\text { Quench } \\
\text { Corrected }\end{array}$} & \multicolumn{2}{|c|}{$\begin{array}{l}\text { Efficiency } \\
\text { Corrected }\end{array}$} & \\
\hline & CPM 1 & \pm CPM & DPM & $\pm \mathrm{DPM}$ & $\begin{array}{c}\% \text { Bio- } \\
\text { originated }\end{array}$ & Error \\
\hline 20\% Bio 1 & 92.32 & $3.85 \%$ & 114.98 & $4.04 \%$ & 23.2 & $4.0 \%$ \\
\hline 20\% Bio 2 & 92.93 & $3.78 \%$ & 115.74 & $3.98 \%$ & 24.3 & $4.0 \%$ \\
\hline 20\% Bio 3 & 89.35 & $3.80 \%$ & 111.27 & $4.00 \%$ & 17.8 & $4.00 \%$ \\
\hline 20\% Bio 5 & 92.74 & $3.80 \%$ & 115.50 & $3.99 \%$ & 24.0 & $4.0 \%$ \\
\hline 20\% Bio 6 & 92.56 & $3.79 \%$ & 115.27 & $3.99 \%$ & 23.6 & $4.0 \%$ \\
\hline 20\% Bio 7 & 2613.32 & $149.33 \%$ & 3254.58 & $149.33 \%$ & 4602.5 & $149.3 \%$ \\
\hline 20\% Bio 8 & 92.15 & $3.79 \%$ & 114.76 & $3.99 \%$ & 22.9 & $4.0 \%$ \\
\hline 20\% Bio 9 & 92.00 & $3.79 \%$ & 114.57 & $3.99 \%$ & 22.6 & $4.0 \%$ \\
\hline 20\% Bio 10 & 89.29 & $3.83 \%$ & 111.21 & $4.03 \%$ & 17.7 & $4.0 \%$ \\
\hline 100\% Bio 11 & 93.07 & $3.97 \%$ & 115.90 & $4.16 \%$ & 24.5 & $4.2 \%$ \\
\hline
\end{tabular}

\section{Sample Cleanup of Highly Quenched and Dyed Fuel}

To further investigate the highly quenched Bio 7 Fuel sample, additional dilution and sample cleanup to remove quenching ingredients was performed. This sample was the only sample analyzed to be corn-oil-based and it had a deep orange color, which was postulated to be responsible for the high level of quenching (note: the deep orange color is not due to the 
presence of additives such as Solvent Red Dye 164). The bio-originated fuel content of the Bio 7 Fuel sample could not be accurately determined due to extreme quenching, even when diluted to $20 \%$. Therefore, a Bio 7 Fuel sample diluted to $10 \%$ with fossil fuel was prepared and analyzed. The quenching in this very dilute sample was still too intense to allow LSC analysis. In a final attempt to reduce the quenching effect of the Bio 7 Fuel sample, an aliquot was cleaned using SPE to remove the color - this is the Cleaned Bio 7 Fuel sample. Analysis of the Cleaned Bio 7 Fuel sample by GC/FID before and after cleanup confirmed that all of the fuel components in the sample were eluted and collected, see Figure 2 (the major components of the bio-originated fuel are C-18 and C-16 fatty acid methyl esters, typical components of biodiesel). A portion, but not all, of the color was removed by SPE. Figure 3 compares the sample color of the 100\%, 10\% Diluted and Cleaned Bio 7 Fuel samples. After cleanup, the sample was diluted to $20 \%$ with fossil fuel and analyzed - this is the $20 \%$ Cleaned Bio 7 sample. Table 7 compares data for the Bio 7 sample analyzed at different dilutions and after cleaning. While the quenching was only slightly reduced to $96.4 \%$ - which resulted in a large error - it was possible to determine the presence and rough percent bio-originated fuel in the $20 \%$ Cleaned Bio 7 Fuel sample.

Table 7. Comparison of Bio 7 sample results for 100\% Fuel, 20\% Dilute Fuel, 10\% Dilute Fuel and Cleaned Fuel sample preparations.

\begin{tabular}{|c|c|c|c|c|c|c|}
\hline \multirow[b]{2}{*}{ Sample } & \multicolumn{2}{|c|}{$\begin{array}{l}\text { Quench } \\
\text { corrected }\end{array}$} & \multicolumn{2}{|c|}{$\begin{array}{l}\text { Efficiency } \\
\text { Corrected }\end{array}$} & \multicolumn{2}{|c|}{ Calculated Results } \\
\hline & CPM & \pm CPM & DPM & $\pm \mathrm{DPM}$ & $\begin{array}{l}\% \text { Bio- } \\
\text { originated }\end{array}$ & Error \\
\hline 100\% Bio 7 & 5191.68 & $177.07 \%$ & 6465.61 & $177.07 \%$ & 9286.1 & $177.1 \%$ \\
\hline 20\% Bio 7 & 2613.32 & $149.33 \%$ & 3254.58 & $149.33 \%$ & 4602.54 & $149.33 \%$ \\
\hline 10\% Bio 7 & -273.93 & $81.13 \%$ & -341.15 & $81.14 \%$ & -642.8 & $81 \%$ \\
\hline $\begin{array}{l}20 \% \text { Cleaned Bio } \\
7\end{array}$ & 98.62 & $17.05 \%$ & 122.81 & $17.09 \%$ & 34.6 & $17 \%$ \\
\hline
\end{tabular}

The effect of dye in bio-originated fuel on quenching was also investigated. Solvent Red Dye 164 is commonly added to fuel in the United States to distinguish between low-taxed or tax exempt fuel products as required by 26 CFR Part 48. To determine how dye would affect LSC analysis and whether sample cleaning is feasible, a bio-originated fuel sample was infused with approximately $12 \mathrm{mg} / \mathrm{L}$ Solvent Red Dye 164 as measured by ASTM D6258 (Bio 12). In an attempt to remove a significant portion of the Solvent Red Dye from the bio-originated fuel sample without chemical modification or dilution, half of the dyed sample was cleaned by SPE. Figure 4 compares the color of the dyed and cleaned sample. Both the dyed and cleaned samples were analyzed by GC/FID to confirm that fuel components were retained after SPE collected (results similar to Figure 2, data not shown), and by LSC to determine the biooriginated fuel content. The LSC results for the Bio 12 Fuel samples listed in Table 8, demonstrate that reduction in quenching for the SPE cleaned sample is possible and that cleaning does not significantly affect the percent recovery of bio-originated fuel. While the 
red dye did increase quenching, it was still possible to obtain accurate results without cleanup for this sample.

Table 8. Comparison of fuel samples spiked with Solvent Red Dye 164 and subsequently cleaned.

\begin{tabular}{l|cc|cc|cc} 
& \multicolumn{2}{|c|}{$\begin{array}{c}\text { Quench } \\
\text { corrected }\end{array}$} & \multicolumn{2}{c|}{$\begin{array}{c}\text { Efficiency } \\
\text { Corrected }\end{array}$} & \multicolumn{2}{c}{ Calculated Results } \\
Sample & CPM & \pm EPM & DPM & \pm DPM & $\begin{array}{l}\% \text { Bio- } \\
\text { originated }\end{array}$ & Error \\
\hline $100 \%$ Bio 12 & 139.35 & $4.83 \%$ & 173.54 & $4.98 \%$ & 99.3 & $5 \%$ \\
$100 \%$ Cleaned Bio & 137.65 & $4.50 \%$ & 171.43 & $4.67 \%$ & 96.3 & $5 \%$ \\
12 & 92.01 & $4.36 \%$ & 114.59 & $4.53 \%$ & 16.6 & $5 \%$ \\
$20 \%$ Bio 12 & 99.01 & $4.36 \%$ & 123.31 & $4.53 \%$ & 20.6 & $5 \%$ \\
$20 \%$ Cleaned Bio 12 & & & & & & 5
\end{tabular}

\section{Renewable mixtures}

Renewable bio-originated fuel sold in the US is typically clear or lightly colored. Examples of GC/FID analyses of a typical diesel fossil fuel and a renewable bio-originated fuel are shown in Figure 5. The main components in the fossil fuel (top) are n-alkanes. The n-alkanes are also present in the renewable bio-originated fuel (bottom), however, increased concentrations of branched alkanes are present as identified by GC/MS. To investigate the accuracy of the modified direct LSC method for the determination of bio-originated fuel content in commercially available fuel, an experiment was conducted in which a series of blended renewable fuel mixtures in diesel fuel were analyzed. To remove any potential bias, a series of blended renewable mixtures ranging from 5 to 99.5 bio-originated renewable fuel were prepared by one analysist and subsequently analyzed as unknown samples by a second analyst. The difference between the measured vs expected bio-originated fuel content was within $\pm 5 \%$ of the actual value for all samples, Table 10 . In addition, results for samples containing 25,50 , and $75 \%$ renewable fuel prepared using different diesel fossil fuels and different renewables were all within $\pm 4 \%$ indicating that the method is robust when fuels originate from various sources. 
Table 10: Measured vs expected bio-originated fuel content in blended renewable fuel samples.

\begin{tabular}{|c|c|c|c|c|}
\hline Sample $^{a}$ & $\begin{array}{l}\text { \% Bio- } \\
\text { originated } \\
\text { fuel }\end{array}$ & Error & Expected \% & $\begin{array}{l}\text { Difference } \\
\text { (Measured - } \\
\text { Expected) }\end{array}$ \\
\hline Blended 1 & 97.1 & $4 \%$ & 99.5 & -2.4 \\
\hline Blended 2 & 97.0 & $4 \%$ & 99.0 & -2.0 \\
\hline Blended 3 & 93.0 & $4 \%$ & 98.0 & -5.0 \\
\hline Blended 4 & 92.1 & $4 \%$ & 95.0 & -2.9 \\
\hline Blended 5 & 89.7 & $4 \%$ & 90.0 & -0.3 \\
\hline Blended 6 & 76.3 & $4 \%$ & 75.0 & 1.3 \\
\hline Blended 7 & 46.8 & $4 \%$ & 50.0 & -3.2 \\
\hline Blended 8 & 25.3 & $4 \%$ & 25.0 & 0.3 \\
\hline Blended 9 & 8.2 & $4 \%$ & 10.0 & -1.8 \\
\hline Blended 10 & 3.8 & $4 \%$ & 5.0 & -1.2 \\
\hline Blended 11 & 72.7 & $4 \%$ & 75.0 & -2.3 \\
\hline Blended 12 & 47.8 & $4 \%$ & 50.0 & -2.2 \\
\hline Blended 13 & 21.1 & $4 \%$ & 25.0 & -3.9 \\
\hline Blended 14 & 73.5 & $4 \%$ & 75.0 & -1.5 \\
\hline Blended 15 & 47.7 & $4 \%$ & 50.0 & -2.3 \\
\hline Blended 16 & 25.8 & $4 \%$ & 25.0 & 0.8 \\
\hline Fossil 1 & -2.5 & $4 \%$ & 0.0 & -2.5 \\
\hline Fossil 2 & -2.2 & $4 \%$ & 0.0 & -2.2 \\
\hline Renewable & 100.6 & $4 \%$ & 98.0 & 2.6 \\
\hline
\end{tabular}


aBlended 1-10 were made with the same renewable/fossil fuel components; Blended 11-13 were made with a different renewable fuel but the same fossil fuel as 1-10; Blended 14-16 were made with the same renewable fuel as 1-10 but a different fossil fuel.

\section{Conclusion}

The Direct LSC method was modified in order to analyze samples exhibiting quenching effects which appeared to be color related for the samples analyzed - although chemical quenching cannot be ruled out. This Modified Direct LSC method was found to be reasonably accurate in determination of bio-originated fuel content of a variety of fuel samples. The bio-originated fuel content of all samples with less than $43 \%$ quenching was able to be determined within $\pm 5 \%$ of the actual fuel percentage. Diluting samples displaying $<90 \%$ quenching values to $20 \%$ bio-originated fuel allowed bio-originated content to be determined in all but one sample. In the most challenging sample (Bio 7 Fuel), clean-up with a SPE column to remove color reduced the quenching effect. While quenching was still high in this sample, we were able to determine presence and rough percent of bio-originated fuel in the sample. Presumably, more effective cleaning methods could lead to higher accuracy in determination of bio-originated fuel in highly quenched samples.

The Modified Direct LSC method was shown to be a good candidate for determination biooriginated fuel content since commercially available renewable fuels are not highly colored and should have little interference due to quenching effects. To investigate the application of this method for renewable fuel, the bio-originated fuel content in 16 samples containing varying concentrations of commercially available renewable fuel from various sources was analyzed and the bio-originated fuel content was determined with an accuracy of $\pm 5 \%$.

\section{Acknowledgements}

This work was supported by the Internal Revenue Service (IRS) under an Interagency Agreement with the U.S. Department of Energy (DOE) under Contract DE-AC05-76RLO 1830. The views, opinions, and findings contained within this report are those of the authors and should not be construed as an official position, policy, or decision of the DOE or IRS unless designated by other documentation. The authors thank Tucker D. Gilman for preparation of renewable fuel blends, sample cleanup, and gas chromatographic analyses.

\section{References}

R. Kristof, M. Hirsch, J. L. Logar, Implementation of the direct LSC method for diesel samples on the fuel market (2014), Applied Radiation and Isotopes, Vol 93, 104-105.

R. Kristof, J. L. Logar, Direct LSC method of biofuels in fuel (2013), Talanta 111, 183-188.

R. Kristof, J. L. Logar, Quench as changing parameter in measurement of biodiesel on liquid scintillation counter (2011) Conference Paper 
R. Idoeta, E. Perez, M. Herranz, F. Legarda, Characteristic parameters in the measurement of ${ }^{14} \mathrm{C}$ of biobased diesel fuels by liquid scintillation (2014) Applied Radiation and Isotopes, Vol 93, 110-113.

G. A. Norton, A. M. Cline, G. C. Thompson, Use of radiocarbon analyses for determining levels of biodiesel in fuel blends - Comparison with ASTM Method D7371 for FAME (2012), Fuel, Vol 96, 284-290.

G. A. Norton, M. X. Woodruff, Simplified Radiocarbon Analysis Procedure for Measuring the Renewable Diesel Concentration in Diesel Fuel Blends (2012), Journal of the American Oil Chemists' Society, 89, 797-803.

I. J. Dijs, E. van der Windt, L. Kaihola, K. van der Borg, Quantitative Determination by ${ }^{14} \mathrm{C}$ Analysis of the Biological Component in Fuels (2006), Radiocarbon, Vol. 48, 315-323.

Perkin Elmer, Differentiation between Fossil and Biofuels (2009) Application Note

C. Varlam, G. Ionita, I. Stefanescu, D. Steflea, Comparative Study Between External Standard Method and Internal Standard Method for Low-Level Tritium Measurements (2001) Nuclear Energy in Central Europe 2001, Portoroz Slovenia, September 10-13, 2001

E. Minne, F. Heynen, C. Delporte, S. Hallez, Effect on ${ }^{90} \mathrm{Sr}$ Determination resulting from a possible over-estimation of the external standard quench parameter on Quantulus 1220, (2009) Arizona Board of Reagents

J. Pawlyta, A. Pazdur, A. Z. Rakowski, Commissioning of a Quantulus 1220 Liquid Scintillation Beta Spectrometer for measuring ${ }^{14} \mathrm{C}$ and ${ }^{3} \mathrm{H}$ at natural abundance levels (1998), Radiocarbon, Vol. 40, 201-209. 


\section{Figure Captions}

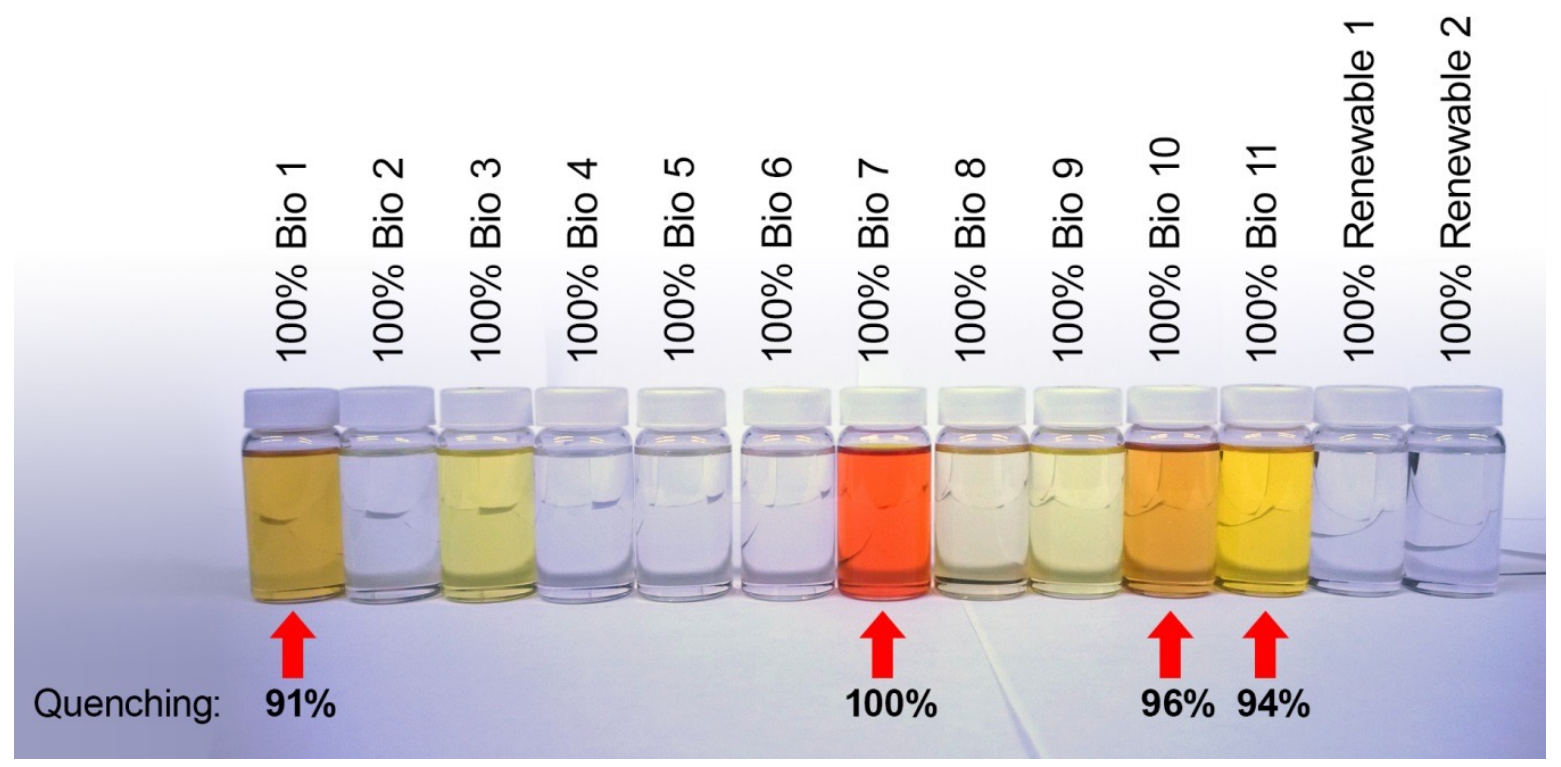

Figure 1. Color of $100 \%$ bio-originated fuel samples prepared for LSC analysis. Arrows indicate samples that had excessive quenching. 


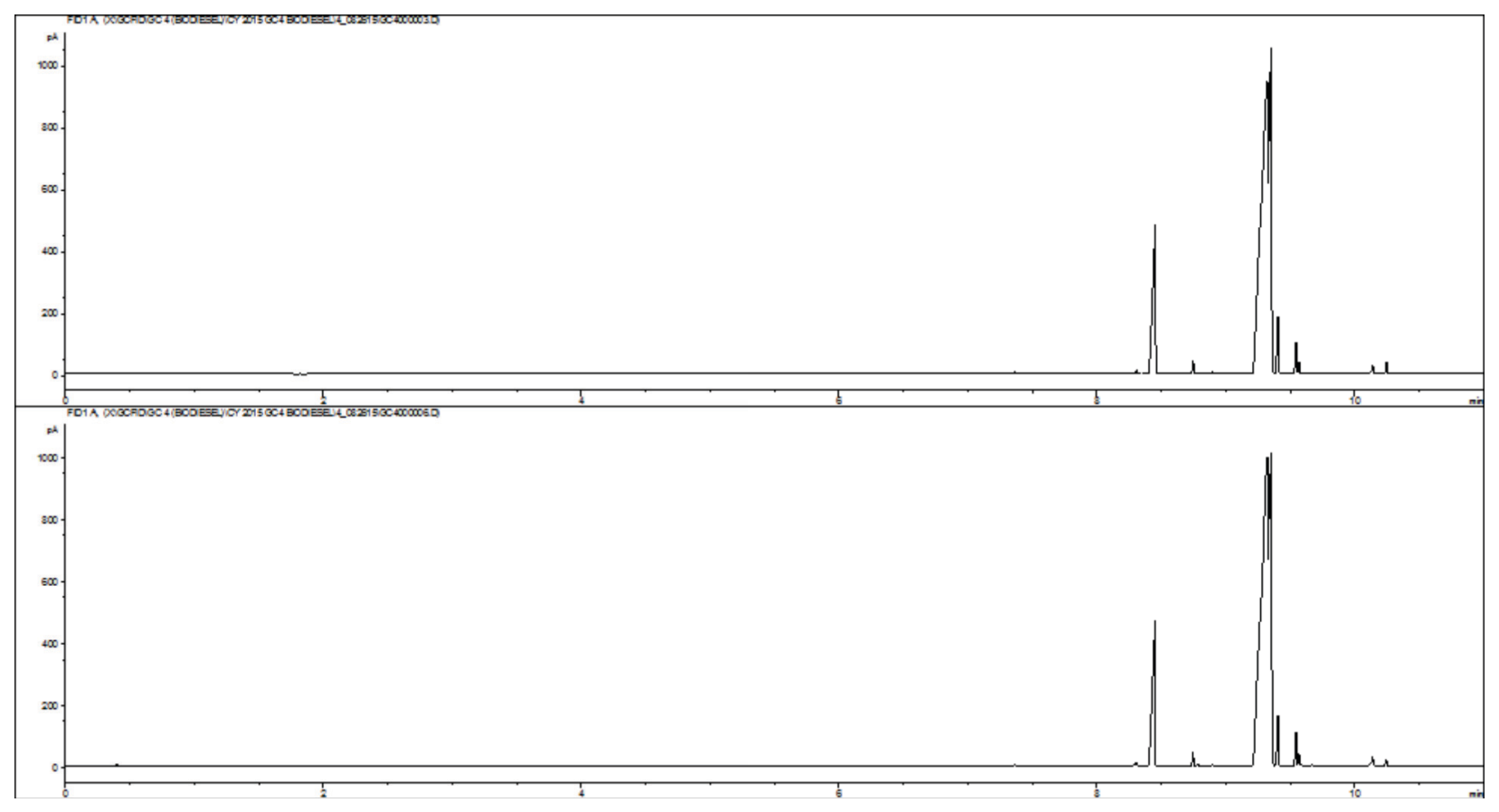

Figure 2. Gas chromatograms for Bio 7 before (top) and after (bottom) sample cleanup to remove color.

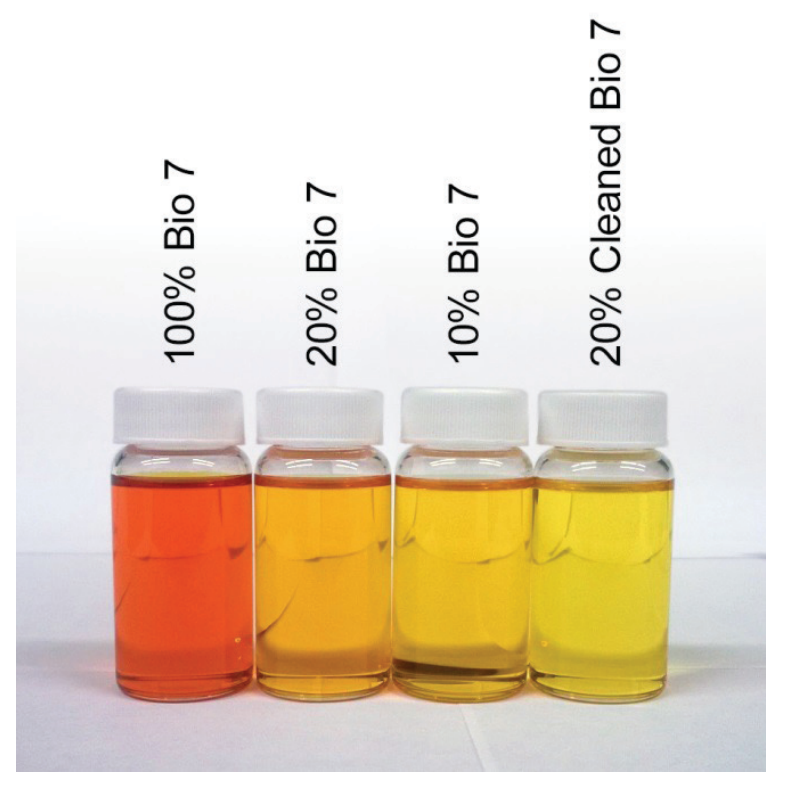

Figure 3. Comparison of color in the Bio 7 100\% Fuel, 20\% Diluted and 10\% Diluted samples along with the $20 \%$ Cleaned Bio 7 Fuel samples. 


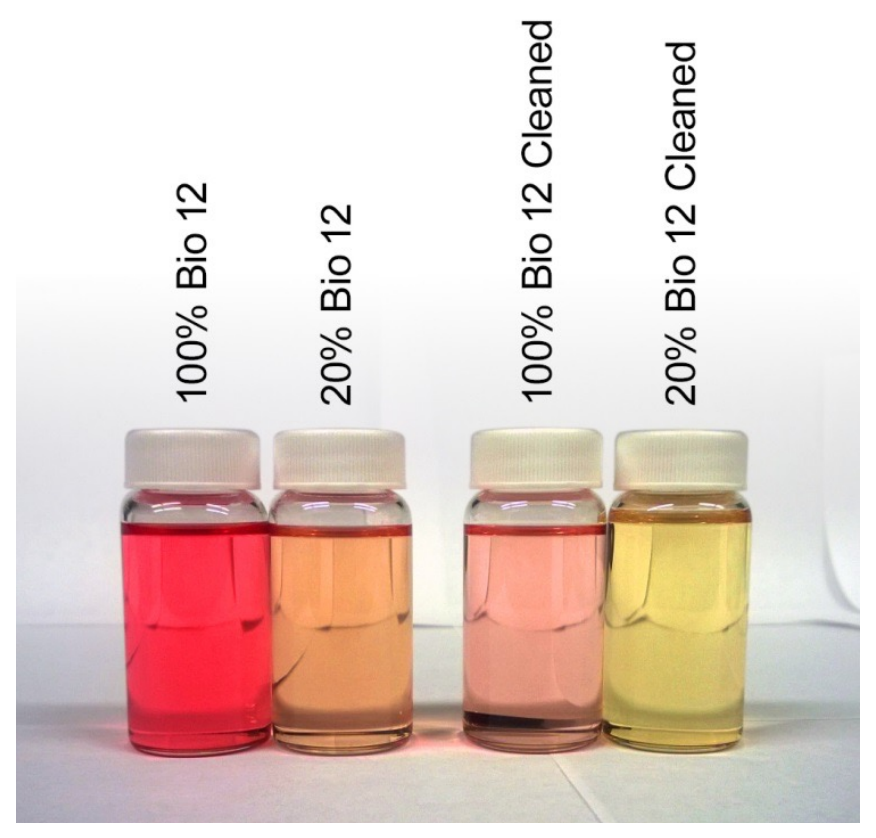

Figure 4. Color comparison of Bio 12 Fuel samples before and after cleaning to remove dye.

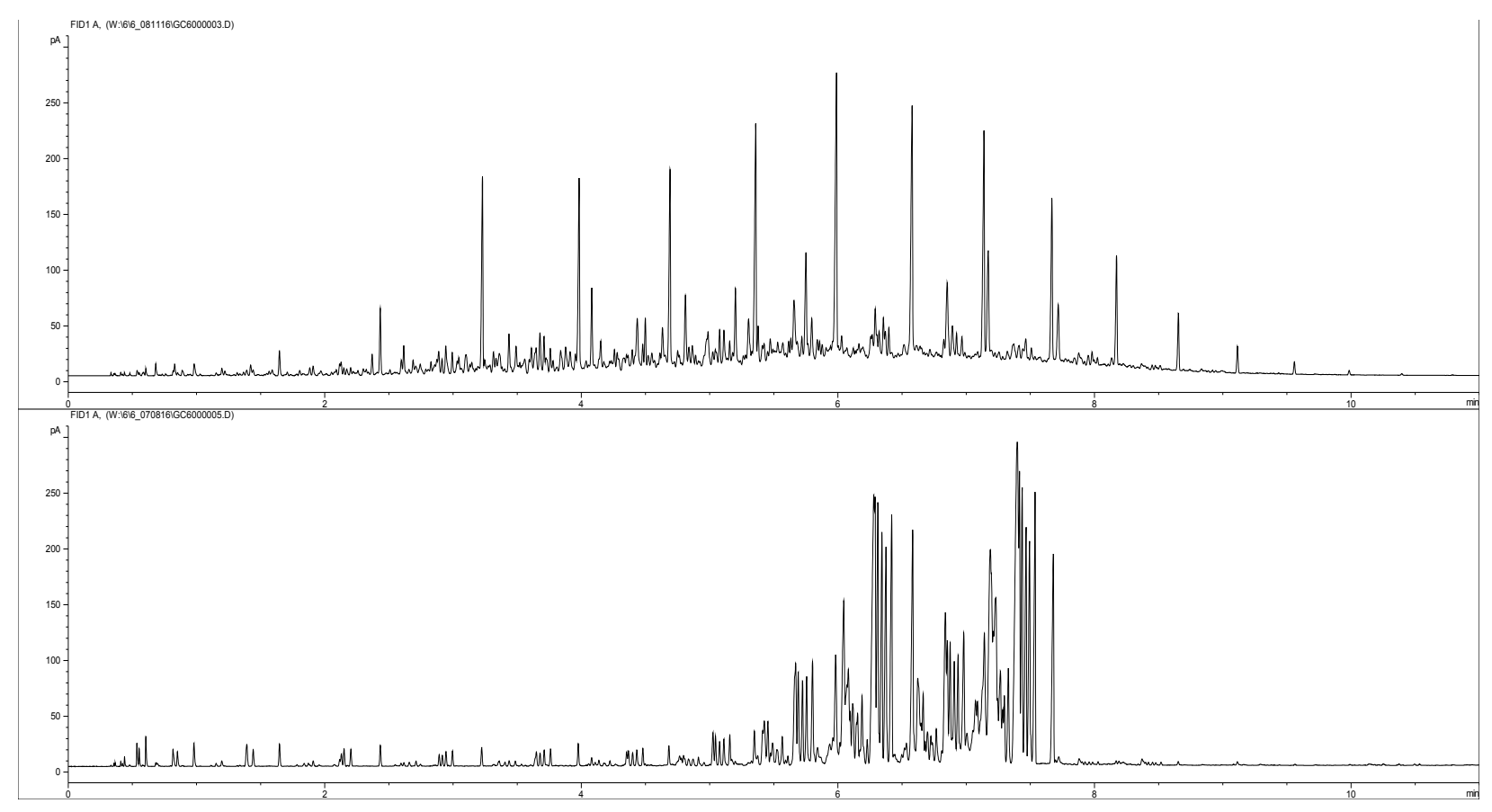

Figure 5. Example gas chromatograms for a typical No. 2 diesel product (Fossil 1, top) and a renewable bio-originated fuel (Renewable 1 , bottom). 